\title{
Peak of Standardized Uptake Value in Oral Cancer Predicts Survival Adjusting for Pathological Stage
}

\author{
HIDENORI SUZUKI ${ }^{1}$, TSUNEO TAMAKI ${ }^{2}$, MASAMI NISHIO ${ }^{3}$, SHINTARO BEPPU $^{1}$, NOBUAKI MUKOYAMA ${ }^{4}$, \\ NOBUHIRO HANAI ${ }^{1}$, DAISUKE NISHIKAWA ${ }^{1}$, YUSUKE KOIDE ${ }^{1}$ and YASUHISA HASEGAWA ${ }^{1}$ \\ ${ }^{1}$ Department of Head and Neck Surgery, Aichi Cancer Center Hospital, Nagoya, Japan; \\ ${ }^{2}$ Department of East Nagoya Imaging Diagnosis Center, Nagoya, Japan; \\ ${ }^{3}$ Department of Radiology, Nagoya Positron Emission Tomography Imaging Center, Nagoya, Japan; \\ ${ }^{4}$ Deparment of Othorhinolaryngology, Nagoya University Graduate School of Medicine, Nagoya, Japan
}

\begin{abstract}
Background/Aim: To predict survival outcomes of different patients with the same stage of disease is difficult. The possible correlation between ${ }^{18} F$-fluorodeoxyglucose $\left({ }^{18} F-F D G\right)$ uptake parameters and survival outcomes was investigated in oral squamous cell carcinoma patients by multivariate analysis adjusted for the pathological stage according to the 8th edition of the tumor-node-metastasis (TNM) classification of the Union for International Cancer Contro. Patients and Methods: ${ }^{18}$ F-FDG-uptake parameters of 28 patients were assessed by positron emission tomography with computed tomography (PET/CT). Results: A peak of standardized uptake value of primary tumor ( $p$-SUVpeak) of $\geq 14.1$ was significantly correlated with shorter overall survival by univariate and multivariate analyses adjusted for the pathological TNM stage. A p-SUVpeak of $\geq 14.1$ was significantly associated with shorter local recurrence-free survival and disease-free survival. Conclusion: A higher p-SUVpeak on pretreatment ${ }^{18} \mathrm{~F}-\mathrm{FDG}$-PET/CT is a prognostic parameter of identifying lower survival outcomes.
\end{abstract}

The tumor-node-metastasis (TNM) staging system is applied worldwide as a predictor of various carcinomas, including oral squamous cell carcinoma (OSCC), but it is difficult to predict survival outcomes of different patients with the same stage (1, 2). Parameters of ${ }^{18} \mathrm{~F}$-2-deoxyglucose $\left({ }^{18} \mathrm{~F}\right.$-FDG) uptake, as assessed by positron emission tomography with computed

This article is freely accessible online.

Correspondence to: Hidenori Suzuki, Department of Head and Neck Surgery, Aichi Cancer Center Hospital, Nagoya, Aichi 4648681, Japan. Tel: +81 527646111, Fax: +81 527642944, e-mail: hi.suzuki@aichi-cc.jp.

Key Words: Oral squamous cell carcinoma, ${ }^{18} \mathrm{~F}-\mathrm{FDG}-\mathrm{PET} / \mathrm{CT}$, SUVpeak, overall survival, UICC8th. tomography (PET/CT), in head and neck cancer, were shown to be associated with overall survival (OS) by multivariate analysis adjusted for TNM stage of the 7th edition of the Union for International Cancer Control (UICC7th) $(3,4)$. However, no study has yet investigated the association between survival outcomes and ${ }^{18} \mathrm{~F}$-FDG-uptake parameters in OSCC by multivariate analysis adjusted for TNM stage based on the 8th edition of the Union for International Cancer Control (UICC8th) published in 2017 (1).

In OSCC, the presence of pathological lymph node metastasis $(\mathrm{pN}+)$ is the most classical predictor, and the presence of extranodal extension (ENE+) is widely accepted as being predictive of poor survival $(5,6)$. The maximum standardized uptake value (SUVmax), which is commonly used as ${ }^{18} \mathrm{~F}$-FDG-uptake parameter, was reported to predict the risk of both $\mathrm{pN}+$ and ENE+ $(7,8)$. However, usefulness of volumetric ${ }^{18} \mathrm{~F}$-FDG-uptake parameters, such as metabolic tumor volume (MTV), total legion glycolysis (TLG), peak of standardized uptake value (SUVpeak), to predict both $\mathrm{pN}+$ and ENE+ remains unknown.

Therefore, the aim of this study was to identify associations between ${ }^{18} \mathrm{~F}$-FDG-uptake parameters and survival outcomes in OSCC by univariate and multivariate analyses adjusted for the pathological TNM stage based on UICC8th, and determine whether ${ }^{18} \mathrm{~F}$-FDG-uptake parameters, including volumetric parameters, are correlated with either $\mathrm{pN}+$ or ENE+.

\section{Patients and Methods}

Patients and clinicopathological parameters. Both primary tumor surgery and neck dissection without preoperative treatment underwent in 50 patients with newly diagnosed OSCC with clinical lymph node metastasis at the Aichi Cancer Center Hospital between January 2008 and July 2013, as previously described (2, 9). Among these 50 patients, 28 received pretreatment 18 F-FDG-PET/CT at East Nagoya Imaging Diagnosis Center were enrolled in this study. The study was approved by the Institutional Review Board, and informed consent was obtained from each patient prior to 
in vivo $32: 1193-1198(2018)$

Table I. Clinicopathological parameters of 28 patients with oral squamous cell carcinoma.

\begin{tabular}{llc}
\hline Parameter & & Number \\
\hline Age & Mean \pm standard deviation (year) & $61.1 \pm 14.9$ \\
Gender & Male/Female & $16 / 12$ \\
Primary tumor site & Tongue/Others & $19 / 9$ \\
Type of neck dissection & Unilateral/Bilateral & $19 / 9$ \\
Type of postoperative therapy & Chemoradiation/Radiation/Chemotherapy/None & $5 / 4 / 2 / 17$ \\
Pathological T classification (UICC7th) & T1/T2/T3/T4a/T4b & $3 / 13 / 7 / 5 / 0$ \\
Pathological N classification (UICC7th) & N0/N1/N2a/N2b/N2c/N3 & $7 / 3 / 0 / 17 / 1 / 0$ \\
Pathological stage (UICC7th) & I/II/III/IVA/IVB & $1 / 4 / 3 / 20 / 0$ \\
Positive surgical margin & Presence/Absence & $5 / 23$ \\
ENE & ENE+/ENE- & $9 / 19$ \\
Positive surgical margin and/or ENE+ & Presence/Absence & $12 / 16$ \\
Size & Mean \pm standard deviation (mm) & $33.8 \pm 11.7$ \\
Tumor thickness & Mean \pm standard deviation (mm) & $16.8 \pm 7.4$ \\
Depth of invasion & Mean \pm standard deviation (mm) & $14.3 \pm 9.32$ \\
Pathological tumor volume & Mean \pm standard deviation $(\mathrm{cm} 3$ ) & $8.87 \pm 9.06$ \\
Lymph node density & Mean \pm standard deviation & $0.06 \pm 0.06$ \\
Pathological T classification (UICC8th) & T1/T2/T3/T4a/T4b & $1 / 2 / 20 / 5 / 0$ \\
Pathological N classification (UICC8th) & N0/N1/N2a/N2b/N2c/N3a/N3b & $7 / 2 / 1 / 10 / 0 / 8$ \\
Pathological stage (UICC8th) & I/II/III/IVA/IVB & $0 / 1 / 6 / 13 / 8$
\end{tabular}

OSCC: Oral squamous cell carcinoma; UICC7th: the 7th edition of Union for International Cancer Control; ENE: extranodal extension; UICC8th: the 8th edition of Union for International Cancer Control.

treatment examination. The procedures of initial TNM staging based on UICC7th, treatments, pathological examinations, followup as well as the assessments of pathological parameters for tumor size and thickness, pathological tumor volume (PTV), lymph node density are described elsewhere $(2,9,10)$. Depth of invasion was assessed from deepest point of invasion to horizon of the adjacent mucosal basement membrane according to AJCC8th staging manual (11). Restaging was performed both from pathological reports and depth of invasion based on UICC8th, as described by Sano (12). The clinicopathological parameters are listed in Table I.

Image assessment. The procedures of both ${ }^{18} \mathrm{~F}-\mathrm{FDG}-\mathrm{PET} / \mathrm{CT}$ scan (Biograph True Point PET/CT/40 with True V: Siemens Health Medical Solutions Inc., Malvern, PA, USA) and semiquantitative evaluation for ${ }^{18} \mathrm{~F}-\mathrm{FDG}$-uptake parameters (Advantage Workstation 4.6 software program the PET VCAR: GE Healthcare, Chalfont, UK) were described previously (10). The mean \pm standard deviation (SD) of blood sugar level at staging was $114.0 \pm 34.3 \mathrm{mg} / \mathrm{dl}$, and the mean \pm SD duration from ${ }^{18}$ F-FDG-PET/CT to surgery was $19.1 \pm 9.1$ days. The SUVmax, SUVpeak, MTV, TLG of the primary tumor were defined as p-SUVmax, p-SUVpeak, p-MTV, p-TLG, respectively. The p-MTV and p-TLG were calculated by adopting a threshold fraction of $45 \%$ of the p-SUVmax from the volumetric region of interest on three-dimensional images. The p-SUVpeak was calculated as the average standardized uptake value within a $1-\mathrm{cm}^{3}$ spherical volumetric region of interest that included the maximum number of pixels.

Diagnosis of $p N+$ and $E N E+$. The ${ }^{18} \mathrm{~F}-\mathrm{FDG}$-uptake parameters of lymph nodes with $\mathrm{pN}+$ and/or ENE+ were reassessed based on a report by Dequanter et al. (7) with minor modifications. Regions of
$\mathrm{pN}+$ and/or ENE+ were recorded in reference to the system of the Japan Neck dissection Study Group and classified based on submental and submandibular, jugular, or posterior triangle lymph nodes (5). A site of abnormal accumulation of ${ }^{18} \mathrm{~F}-\mathrm{FDG}$ on PET/CT was interpreted as the presence of lymph node metastasis, while normal accumulation was considered as metastasis-free. The SUVmax, SUVpeak, MTV, TLG of the lymph node were defined as ln-SUVmax, ln-SUVpeak, ln-MTV, ln-TLG, respectively. For the semiquantitative analysis, the ${ }^{18} \mathrm{~F}$-FDG-uptake parameters of the lymph node of interest, which included ln-SUVmax, ln-SUVpeak, ln-MTV, ln-TLG, were measured from the site of abnormal accumulation. If there were multiple lymph nodes within a certain region, the node with the highest ln-SUVmax was selected. At each region, all PET/CT results were compared with pathological results, which were considered as gold standard.

Statistical analysis. Statistical analyses were performed using JMP software (version 9; SAS Institute: Cary, NC, USA). Simple regression analysis was used to identify correlations between PTV and ${ }^{18}$ F-FDG-uptake parameters of primary tumor. OS was defined as the duration from FDG-PET/CT to death or last contact and assessed by Kaplan-Meier method. OS based on pathological stage of UICC8th (I-IVA, IVB) were compared by log-rank test. The correlations between OS and the cut-off values for various ${ }^{18} \mathrm{~F}$ FDG-uptake parameters of the primary tumor were identified by log-rank test $(2,9)$. Multivariate analysis adjusting pathological stage of UICC8th (IVB; I-IVA) was performed to identify correlations between ${ }^{18} \mathrm{~F}-\mathrm{FDG}$-uptake parameters of primary tumor and OS by a Cox's proportional hazards model. The relationships between p-SUVpeak groups $(\geq 14.1 ;<14.1)$ and clinicopathological parameters were assessed by Mann-Whitney $U$ - or chi-squared test. The Kaplan-Meier method was used to identify differences between 


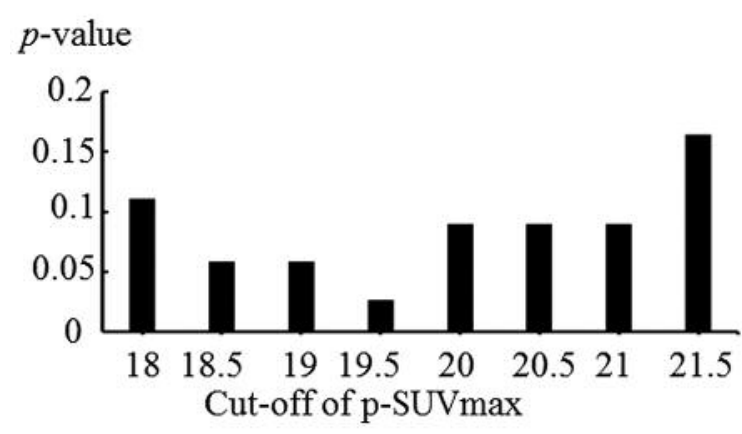

$p$-value
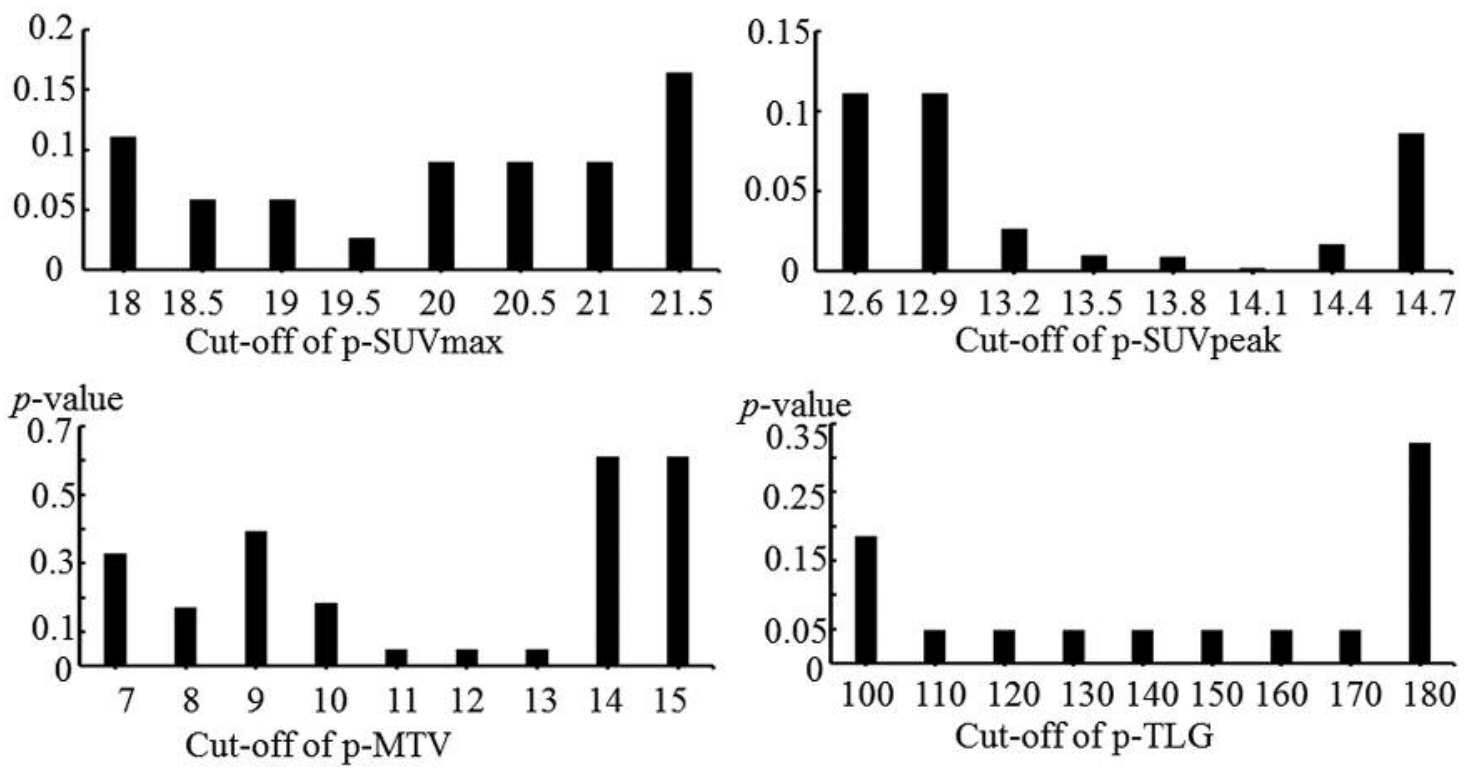

Figure 1. Probability values of OS analysis using different cut-off levels for p-SUVmax, p-SUVpeak, p-MTV, p-TLG values. OS: Overall survival; p-SUVmax: maximum standardized uptake value of primary tumor; p-SUVpeak: peak standardized uptake value of primary tumor; p-MTV: metabolic tumor volume of primary tumor; $p$-TLG: total lesion glycolysis of primary tumor.

the p-SUVpeak groups in local recurrence-free survival (LRFS), regional recurrence-free survival (RRFS), distant metastasis-free survival (DMFS), disease-free survival (DFS). The area under the curve (AUC) of receiver-operating characteristic curve, sensitivity, 1 -specificity were calculated to detect the best cut-off values for ${ }^{18} \mathrm{FDG}$-uptake parameters of lymph node compare $\mathrm{pN}+$ versus pathological lymph node metastasis (pN-), and ENE+ versus ENEby logistic regression analysis. $p<0.05$ was considered statistically significant.

\section{Results}

PTV and ${ }^{18}$ F-FDG-uptake parameters. Among all patients, the mean \pm SD values of PTV, p-SUVmax, p-SUVpeak, p-MTV, p-TLG were $8.87 \pm 9.06 \mathrm{~cm}^{3}, 17.8 \pm 6.62 \mathrm{~g} / \mathrm{ml}, 12.1 \pm 4.62 \mathrm{~g} / \mathrm{ml}$, $5.64 \pm 4.70 \mathrm{~cm}^{3}, 66.1 \pm 61.0 \mathrm{~g}$, respectively. PTV was significantly correlated with both p-MTV $(p<0.01)$ and p-TLG $(p<0.01)$, but not with either $\mathrm{p}-\mathrm{SUVmax}$ or $\mathrm{p}-\mathrm{SUV}$ peak.

Clinical course. The mean \pm SD duration of follow-up among the whole population, the 18 patients who survived, the 10 patients who died was $45.3 \pm 27.0,62.1 \pm 17.0,15.2 \pm 8.23$ months, respectively. The 3-year rates of OS, LRFS, RRFS, DMFS, DFS were $64.3 \%, 78.3 \%, 71.1 \%, 76.5 \%, 63.8 \%$, respectively. Pathological stage IVB of UICC8th was significantly correlated with shorter OS than pathological stage I-IVA of UICC8th $(p=0.01)$.
Cut-off values of ${ }^{18} F$-FDG-uptake parameters. The lowest $\mathrm{p}$ values were $\mathrm{p}$-SUVmax $=19.5(p=0.03), \mathrm{p}-\mathrm{SUV}$ peak $=14.1$ $(p<0.01), \mathrm{p}-\mathrm{MTV}=11 \quad(p=0.049), \mathrm{p}-\mathrm{TLG}=110 \quad(p=0.049)$. (Figure 1).

Multivariate OS analysis. Multivariate analysis adjusted for ${ }^{18} \mathrm{~F}$-FDG-uptake parameters and pathological stage of UICC8th, p-SUVpeak $\geq 14.1 \quad(p=0.047)$ was significantly associated with shorter OS, while p-SUVmax of $\geq 19.5$, p$\mathrm{MTV} \geq 11, \mathrm{p}-\mathrm{TLG} \geq 110$ were not (Table II).

The p-SUVpeak groups. The relations between clinicopathological parameters of the two groups (pSUVpeak $\geq 14.1 ;<14.1$ ) are shown in Table III. A pSUVpeak $\geq 14.1$ was significantly associated with larger tumor size $(p=0.04)$, greater $\mathrm{p}-\mathrm{SUV} \max (p<0.01)$ and $\mathrm{p}$ TLG $(p=0.02)$. A $p$-SUVpeak $\geq 14.1$ was significantly associated with shorter LRFS $(p=0.02)$ and DFS $(p<0.01)$. However, there were no significant differences in RRFS or DMFS. The Kaplan-Meier curves are shown in Figure 2.

$p N+$ and $E N E+$. The relations between the ${ }^{18} \mathrm{~F}-\mathrm{FDG}$-uptake parameters and pathological findings were assessed in 88 cervical regions. The numbers of regions classified as $\mathrm{pN}+$ and ENE+ totaled 31 and 11, respectively. The numbers of regions not classified as $\mathrm{pN}+$ and $\mathrm{ENE}+$ totaled 57 and 77, 
in vivo $32: 1193-1198(2018)$

Table II. Multivariate analysis of overall survival by a Cox's proportional hazards model.

\begin{tabular}{|c|c|c|c|c|}
\hline \multicolumn{2}{|l|}{ Characteristic } & \multirow{2}{*}{$\frac{\text { Hazards ratio }}{3.09}$} & \multirow{2}{*}{$\frac{95 \% \text { confidence interval }}{0.83-12.84}$} & \multirow{2}{*}{$\frac{p \text {-Value }}{0.09}$} \\
\hline Model 1 Pathological stage (UICC 8th) & IVB/I-IVA & & & \\
\hline p-SUVmax & $\geq 19.5 /<19.5$ & 2.96 & $0.77-14.44$ & 0.12 \\
\hline Model 2 Pathological stage (UICC 8th) & IVB/I-IVA & 2.31 & $0.10-1.76$ & 0.24 \\
\hline p-SUVpeak & $\geq 14.1 /<14.1$ & 4.16 & $1.02-18.88$ & 0.047 \\
\hline Model 3 Pathological stage (UICC 8th) & IVB/I-IVA & 3.54 & $0.94-14.63$ & 0.06 \\
\hline p-MTV & $\geq 11 /<11$ & 2.29 & $0.47-8.93$ & 0.28 \\
\hline Model 4 Pathological stage (UICC 8th) & IVB/I-IVA & 3.54 & $0.94-14.63$ & 0.06 \\
\hline p-TLG & $\geq 110 /<110$ & 2.29 & $0.47-8.93$ & 0.28 \\
\hline
\end{tabular}

UICC8th: The 8th edition of Union for International Cancer Control; p-SUVmax: maximum of standardized uptake value of primary tumor; pSUVpeak: peak of standardized uptake value of primary tumor; p-MTV: metabolic tumor volume of primary tumor; p-TLG: total lesion glycolysis of primary tumor.

Table III. Relationships between clinicopathological parameters and p-SUVpeak $(<14.11 \geq 14.1)$.

\begin{tabular}{|c|c|c|c|c|}
\hline \multirow[t]{2}{*}{ Parameter } & & \multicolumn{2}{|c|}{ p-SUVpeak } & \multirow[t]{2}{*}{$p$-Value } \\
\hline & & $<14.1(\mathrm{n}=20)$ & $\geq 14.1(\mathrm{n}=8)$ & \\
\hline Age & Mean \pm S.D. & $58.1 \pm 15.5$ & $68.5 \pm 10.6$ & $0.13^{\dagger}$ \\
\hline Gender & Male/Female & $9 / 11$ & $7 / 1$ & $0.09 *$ \\
\hline Primary tumor site & Tongue/Others & $14 / 6$ & $5 / 3$ & $1.00 *$ \\
\hline Type of neck dissection & Unilateral/Bilateral & $13 / 7$ & $6 / 2$ & $1.00 *$ \\
\hline Type of postoperative therapy & Presence/Absence & $7 / 13$ & $4 / 4$ & $0.67 *$ \\
\hline Pathological T classification (UICC7th) & $\mathrm{T} 1-\mathrm{T} 2 / \mathrm{T} 3-\mathrm{T} 4$ & $13 / 7$ & $3 / 5$ & $0.23 *$ \\
\hline Pathological N classification (UICC7th) & $\mathrm{N} 0 / \mathrm{N} 1-\mathrm{N} 2$ & $6 / 14$ & $1 / 7$ & $0.63 *$ \\
\hline Pathological stage (UICC7th) & I-III/IV & $7 / 13$ & $1 / 7$ & $0.37 *$ \\
\hline Positive surgical margin & Presence/Absence & $4 / 16$ & $1 / 7$ & $1.00 *$ \\
\hline Extracapsular spread & Presence/Absence & $5 / 15$ & $4 / 4$ & $0.37 *$ \\
\hline Positive surgical margin, or extracapsular spread, or both & Presence/Absence & $8 / 12$ & $4 / 4$ & $0.69 *$ \\
\hline Lymph node density & $\geq 0.06 /<0.06$ & $8 / 12$ & $4 / 4$ & $0.69 *$ \\
\hline Pathological T classification (UICC8th) & $\mathrm{T} 1-\mathrm{T} 3 / \mathrm{T} 4 \mathrm{a}-\mathrm{T} 4 \mathrm{~b}$ & $16 / 4$ & $7 / 1$ & $1.00 *$ \\
\hline Pathological N classification (UICC8th) & $\mathrm{N} 0-\mathrm{N} 1 / \mathrm{N} 2-\mathrm{N} 3 \mathrm{~b}$ & $7 / 13$ & $2 / 6$ & $1.00^{*}$ \\
\hline Pathological stage (UICC8th) & I-III/IVA-IVB & $6 / 14$ & $1 / 7$ & $0.63^{*}$ \\
\hline Size & Mean \pm S.D. & $31.0 \pm 10.2$ & $41.0 \pm 12.5$ & $0.04^{\dagger}$ \\
\hline Depth of invasion & Mean \pm S.D. & $14.4 \pm 9.1$ & $14.0 \pm 10.5$ & $0.84^{\dagger}$ \\
\hline Tumor thickness & Mean \pm S.D. & $16.4 \pm 7.1$ & $17.7 \pm 8.7$ & $0.96^{\dagger}$ \\
\hline Pathological tumor volume & Mean \pm S.D. & $7658 \pm 7743$ & $11903 \pm 11790$ & $0.51^{\dagger}$ \\
\hline p-SUVmax & Mean \pm S.D. & $15.0 \pm 5.2$ & $24.9 \pm 4.2$ & $<0.01^{\dagger}$ \\
\hline p-MTV & Mean \pm S.D. & $4.9 \pm 3.9$ & $7.6 \pm 6.1$ & $0.32^{\dagger}$ \\
\hline $\mathrm{p}$-TLG & Mean \pm S.D. & $48.6 \pm 45.3$ & $109.7 \pm 75.8$ & $0.02^{\dagger}$ \\
\hline
\end{tabular}

p-SUVpeak: Peak of standardized uptake value of primary tumor; UICC7th, the 7th edition of Union for International Cancer Control; UICC8th: the 8th edition of Union for International Cancer Control; Control; p-SUVmax: maximum of standardized uptake value of primary tumor; p-MTV: metabolic tumor volume of primary tumor; p-TLG: total lesion glycolysis of primary tumor. ${ }^{*}$ Chi-squared test or ${ }^{\dagger}$ Mann-Whitney $U$-test.

respectively. The best cut-off values to detect $\mathrm{pN}+$ were $\mathrm{ln}$ SUVmax $=3.33(p<0.01$, AUC $=0.84)$, ln-SUVpeak $=1.82$ $(p<0.01, \mathrm{AUC}=0.82), \ln -\mathrm{MTV}=0.27(p=0.43, \mathrm{AUC}=0.66), \ln -$ $\mathrm{TLG}=1.03(p<0.01, \mathrm{AUC}=0.74)$. The best cut-off values to detect ENE+ were ln-SUVmax=3.61 $(p<0.01, \mathrm{AUC}=0.80)$, ln-
SUVpeak=1.07 $(p<0.01, \mathrm{AUC}=0.79), \ln -\mathrm{MTV}=0.37(p=0.55$, $\mathrm{AUC}=0.66), \ln -\mathrm{TLG}=3.1 \quad(p=0.23, \mathrm{AUC}=0.74)$. The highest AUC to detect $\mathrm{pN}+$ and ENE+ were $1 n-\mathrm{SUVmax}=3.33$ (sensitivity $=80.7 \%$, specificity $=77.2 \%$ ) and $\ln -\mathrm{SUVmax}=3.61$ (sensitivity $=81.8 \%$, specificity $=67.5 \%)$, respectively. 


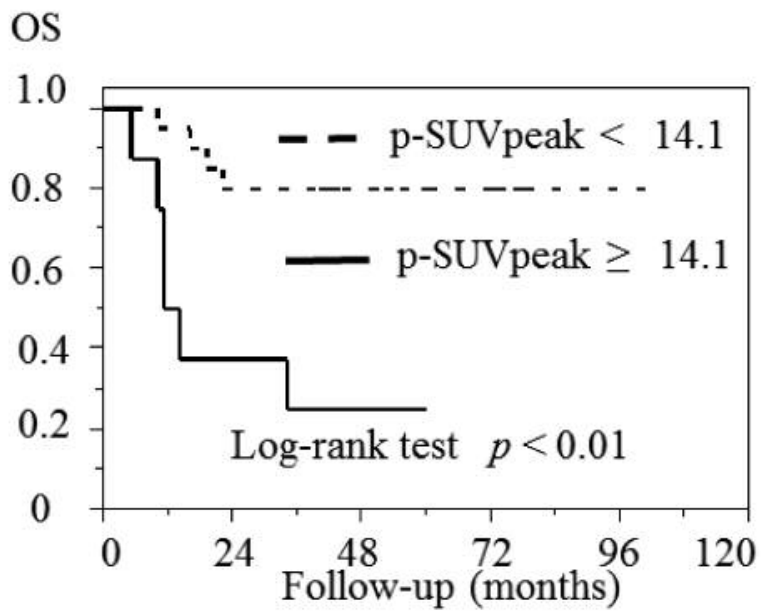

\section{LRFS}

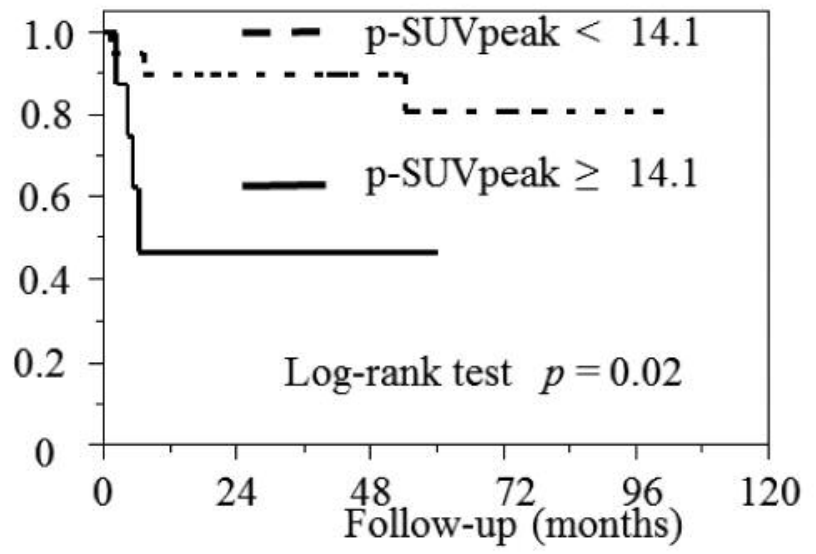

DFS

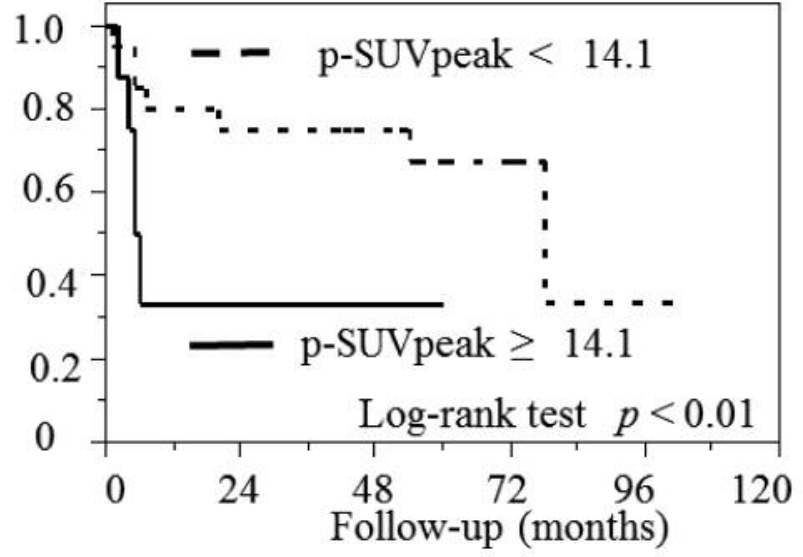

Figure 2. Pathological stage of IVB based on the UICC8th was associated with significantly shorter OS ( $p=0.01)$, and $p$-SUVpeak of $\geq 14.1$ was associated with significantly lower OS $(p<0.01)$, LRFS $(p=0.02)$, DFS $(p<0.01)$. UICC8th: The 8th edition of the Union for International Cancer Control, $p$ SUVpeak: peak standardized uptake value of primary tumor; OS: overall survival; LRFS: local recurrence-free survival; DFS: disease-free survival.

\section{Discussion}

The present study showed, for the first time, significant associations between high p-SUVpeak values and lower OS in OSCC by using multivariate analysis adjusted for pathological stage based on UICC8th. Also, high p-SUVpeak was significantly correlated with reduced LRFS and DFS rates.

In head and neck cancer, including OSCC ${ }^{18}$ F-FDG-uptake parameters were significantly correlated with OS $(3,4,10)$. For example, high p-SUVpeak was predictive of shorter OS in OSCC by multivariate analysis adjusting pathological stage of UICC7th (4). Our findings of a significant association between ${ }^{18} \mathrm{~F}$-FDG-uptake parameters and OS are consistent with these studies $(3,4,10)$. Moreover, the present study identified correlations between survival and ${ }^{18} \mathrm{~F}$-FDG uptake parameters in OSCC by multivariate analysis adjusting TNM stage of UICC8th. The present results suggest that pretreatment SUVpeak is a predictor of shorter OS.

The present findings also demonstrated that greater tumor size, p-SUVmax, p-MTV, p-TLG, which were poor predictors of OSCC $(4,13,14)$, were significantly correlated with higher p-SUVpeak. Moreover, higher SUVpeak was significantly correlated with worse LRFS and DFS in this study, and we suggest that when SUVpeak is relatively high, more aggressive treatment strategies such as postoperative chemoradiotherapy are needed to improve survival outcomes.

In esophageal cancer, ln-MTV was significantly correlated with $\mathrm{pN}+(15)$. In head and neck cancer, ${ }^{18} \mathrm{~F}$-FDG-uptake was used to evaluate treatment response of lymph node metastasis after chemoradiotherapy (16), and the significant association between ${ }^{18}$ F-FDG-uptake and chemosensitivity for cisplatin was reported (17). To the best of our knowledge, the usefulness 
of volumetric ${ }^{18} \mathrm{~F}$-FDG-uptake parameters to predict $\mathrm{pN}+$ and $\mathrm{ENE}+$ has not been investigated. In this study, ln-SUVmax in OSCC had the highest $\mathrm{AUC}=0.82$ to detect both $\mathrm{pN}+$ and ENE+ than ln-MTV, ln-TLG, ln-SUVpeak.

The limitations of the present study include its retrospective design and relatively small number of subjects; thus, prospective analysis with a larger number of subjects is needed to verify these results.

\section{Conclusion}

A high p-SUVpeak was correlated with shorter OS in OSCC by multivariate analysis adjusting pathological stage of UICC8th.

\section{Conflicts of Interest}

The Authors declare no conflict of interest regarding this study.

\section{Acknowledgements}

This study was supported from JSPS KAKENHI Grant Number of 16K11253.

\section{References}

1 Brierley J, Gospodarowicz MD and Wittekind C: TNM classification of malignant tumors. International union against cancer. 8th ed. Oxford, England: Wiley-Blackwell; 2016.

2 Suzuki H, Beppu S, Hanai N, Hirakawa H and Hasegawa Y: Lymph node density predicts lung metastasis in oral squamous cell carcinoma. Br J Oral Maxillofac Surg 54: 213-218, 2016.

3 Yabuki K, Sano D, Shiono O, Arai Y, Takahashi H, Chiba Y, Tanabe T, Nishinura G, Takahashi M, Taguchi T, Kaneta T, Hata $\mathrm{M}$ and Oridate N: Prognostic significance of metabolic tumor volume in patients with piriform sinus carcinoma treated by radiotherapy with or without concurrent chemotherapy. Head Neck 38: 1666-1671, 2016.

4 Abgral R, Keromnes N, Robin P, Le Roux PY, Bourthis D, Palard X, Rousset J, Valette G, Marianowski R and Salaün PY: Prognostic value of volumetric parameters measured by ${ }^{18} \mathrm{~F}-$ FDG-PET/CT in patients with head and neck squamous cell carcinoma. Eur J Nucl Med Mol Imaging 41: 659-667, 2014.

5 Hasegawa Y and Saikawa M: Update on the classification and nomenclature system for neck dissection: revisions proposed by the Japan Neck Dissection Study Group. Int J Clin Oncol 15: 5$12,2010$.

6 Greenberg JS, Fowler R, Gomez J, Mo V, Roberts D, El Naggar AK and Myers JN: Extent of extracapsular spread: a critical prognosticator in oral tongue cancer. Cancer 97: 1464-70, 2003.

7 Dequanter D, Shahla M, Aubert C, Deniz Y and Lothaire P: Prognostic value of FDG PET/CT in head and neck squamous cell carcinomas. Onco Targets Ther 8: 2279-2283, 2015.
8 Su Z, Duan Z, Pan W, Wu C, Jia Y, Han B and Li C: Predicting extracapsular spread of head and neck cancers using different imaging techniques: a systemic review and meta-analysis. Int $\mathrm{J}$ Oral Maxillofac Surg 45: 413-421, 2016.

9 Mukoyama N, Suzuki H, Hanai N, Sone M and Hasegawa Y: Pathological tumor volume predicts survival outcome in oral squamous cell carcinoma. Oncol Lett, 2018. [in press]

10 Suzuki H, Nishio M, Nakanishi H, Hanai N, Hirakawa H, Kodaira T, Tamaki $\mathrm{T}$ and Hasegawa Y: Impact of total lesion glycolysis measured by ${ }^{18} \mathrm{~F}-\mathrm{FDG}-\mathrm{PET} / \mathrm{CT}$ on overall survival and distant metastasis in hypopharyngeal cancer. Oncol Lett 12: 1493-1500, 2016.

11 Amin BA, Edge SB, Greene FL, Byrd DR, Brookland RK, Washington MK, Gershenwald JE, Compton CC, Hess KR, Sullivan DC, Jessup JM, Brierley JD, Gaspar LE, Schilsky RL, Balch CM, Winchester DP, Asare EA, Madera M, Gress DM and Meyer LR: AJCC cancer staging manual, 8th edn. Springer, New York. 2016.

12 Sano D, Yabuki K, Arai Y, Tanabe T, Chiba Y, Nishimura G, Takahashi H, Yamanaka S and Oridate N: The applicability of new TNM classification for humanpapilloma virus-related oropharyngeal cancer in the 8th edition of the AJCC/UICC TNM staging system in Japan: A single-center study. Auris Nasus Larynx 45: 558-565, 2018.

13 Kim M, Achmad A, Higuchi T, Arisaka Y, Yokoo H, Yokoo S and Tsushima Y: Effects of intratumoral inflammatory process on ${ }^{18}$ F-FDG uptake: pathological and comparative study with ${ }^{18}$ F-fluoro- $\alpha$-methyltyrosine PET/CT in oral squamous cell carcinoma. J Nucl Med 56: 16-21, 2015.

14 Heiduschka G, Virk SA, Palme CE, Ch'ng S, Eliot M, Gupta R and Clark J: Margin to tumor thickness ratio - A predictor of local recurrence and survival in oral squamous cell carcinoma. Oral Oncol 55: 49-54, 2016.

15 I HS, Kim SJ, Kim IJ and Kim K: Predictive value of metabolic tumor volume measured by ${ }^{18} \mathrm{~F}-\mathrm{FDG}$ PET for regional lymph node status in patients with esophageal cancer. Clin Nucl Med 37: 442-446, 2012.

16 Nishimura G, Matsuda H, Taguchi T, Takahashi M, Komatsu M, Sano D, Sakuma N, Arai Y and Takahashi H: Treatment evaluation of metastatic lymph nodes after concurrent chemoradiotherapy in patients with head and neck squamous cell carcinoma. Anticancer Res 32: 595-600, 2012.

17 Suzuki H, Nishio N, Hanai N, Hirakawa H, Tamaki T and Hasegawa Y: Correlation between ${ }^{18} \mathrm{~F}$-FDG-uptake and in vitro chemosensitivity of cisplatin in head and neck cancer. Anticancer Res 35: 1009-1016, 2015. 